Cahiers d'études italiennes

\title{
Techniques de séduction dans le polar italien contemporain
}

Barbara Meazzi

\section{(2) OpenEdition}

\section{Journals}

Édition électronique

URL : http://journals.openedition.org/cei/829

DOI : $10.4000 /$ cei.829

ISSN : 2260-779X

Éditeur

UGA Éditions/Université Grenoble Alpes

\section{Édition imprimée}

Date de publication : 15 septembre 2006

Pagination : 173-184

ISBN : 978-2-84310-086-4

ISSN : 1770-9571

\section{Référence électronique}

Barbara Meazzi, «Techniques de séduction dans le polar italien contemporain », Cahiers d'études italiennes [En ligne], 5 | 2006, mis en ligne le 15 mars 2008, consulté le 28 mars 2021. URL : http:// journals.openedition.org/cei/829; DOI : https://doi.org/10.4000/cei.829 


\title{
TECHNIQUES DE LA SÉDUCTION \\ DANS LE POLAR ITALIEN CONTEMPORAIN
}

\author{
Barbara Meazzi \\ Université de Savoie - Chambéry
}

Avant d'introduire mon sujet, il faut trouver une définition de la séduction qui convienne au genre que j'ai choisi d'explorer, c'est-à-dire le roman policier. La séduction prévoit le détournement du droit chemin, du bien, du devoir, ainsi que le signale le Trésor de la Langue Française ${ }^{1}$. L'étymologie du mot, attesté depuis 1175, possède plusieurs connotations indiquant la trahison et le détournement : la séduction serait une action amenant à commettre une faute d'ordre moral. Bien entendu, le terme renvoie également à de nombreuses implications sensuelles, sexuelles et érotiques dont les femmes sont les objets alors que les hommes - cela arrive pourtant rarement - sont les victimes. La séduction demeure en effet surtout une affaire d'hommes : si la femme ose la séduction, c'est qu' elle est monstrueuse, comme la Lulu de Wedekind.

Le séducteur, tel Sade ou encore Don Juan, est par conséquent un corrupteur de femmes et surtout de jeunes filles ; il possède également la capacité de susciter le désir sans pour autant le satisfaire. Le séducteur a plusieurs techniques à sa disposition, selon le contexte, la situation et l'époque. Les conseils donnés aux hommes pour séduire les femmes, sur les sites Internet les plus disparates, sont nombreux. Généralement, la séduction d'une femme se décompose en sept mouvements : il y a d'abord le repérage, puis l'échange de regards et de sourires ; l'infiltration ; la diversion ; l'avancée fulgurante ; le retrait stratégique momentané évidemment suivi de la capitulation de la femelle, circonvenue avec de belles paroles.

La séduction passe donc par le langage : dans son ouvrage de 1916, Come si seducono le donne, Marinetti insiste sur le fait que la femme hait le langage éhonté des hommes, préférant la rhétorique de la séduction ${ }^{2}$.

1. <http://atilf.atilf.fr/tlfv3.htm>

2. Marinetti F.T., Come si seducono le donne (1916), Florence, Vallecchi, 2003, p. 27. 
Pour aboutir dans la conquête, précise encore Marinetti, il faut avoir toutes les qualités du futuriste italien : un corps svelte, fort, agressif ; une belle chevelure ou une belle calvitie électrique ; une vitalité puissante, une voix mâle, des attitudes oratoires bien développées. Mais d'abord et avant tout, pour séduire, il faut considérer la femme comme une sœur de la mer, du vent, des nuages. Les femmes, écrit Marinetti, "pensano, vogliono, lavorano ; preparano anch'esse il progresso intellettuale dell'umanità $»^{3}$.

L'image que les femmes ont de la séduction masculine ne correspond pas toujours à celle que les hommes se font de leur pouvoir de séduction. Nous en avons un bel exemple dans un récit de Matilde Serao, "La virtù di Checchina » : mariée à un véritable rustre, Checchina se laisse séduire par les mots du beau marquis d'Aragona; le marquis se révèle cependant aussi malotru que l'affreux mari, et la séduction décrite par le narrateur est une affaire finalement plutôt expéditive ${ }^{4}$.

\section{Le roman policier et la séduction esthétique : première hypothèse}

Le roman policier est un genre fondé sur deux types de séductions : la séduction (en vérité occasionnelle) entre les personnages principaux - que je désignerai comme séduction sexuelle et que j'illustrerai plus tard - et la séduction que l'auteur exerce sur le lecteur - que j'appellerai séduction esthétique.

Car le roman policier est une machine à lire. Il s'est produit quelque chose de mystérieux que l'on va essayer d'expliquer rationnellement : de ce fait, l'auteur tient le lecteur en haleine jusqu'au bout. Comme le note Sciascia, le lecteur est d'autant plus effrayé, intrigué, trahi et donc séduit assez facilement par l'auteur qu'il s'approche du polar dans un état de repos intellectuel absolu. Le lecteur est au polar ce que la femme serait à l'homme dans le domaine de la séduction : il se laisse emporter par l'émotion et accepte, par convention, " un ruolo di inferiorità e passività intellettuale $»^{5}$.

3. Ibid., p. 97. Enif Robert ajoutera, dans une lettre à Marinetti, que la définition étymologique de la séduction ne correspond plus à la réalité, parce que la femme fait de son corps ce qu'elle en veut et décide de s'offrir à l'homme seulement si elle le souhaite : Robert E., « Lettera aperta a F.T. Marinetti ", Come si seducono le donne, op. cit., p. 113.

4. Serao M., "La virtù di Checchina » (1883), La virtù delle donne, Cava de’ Tirreni, Avagliano Editore, 1999.

5. Sciascia L., "Breve storia del romanzo poliziesco ", Cruciverba (1983), in Opere 1971-1983, C. Ambroise éd., Milan, Bompiani, 2001, p. 1182. Sur l'importance de l'abandon et de l'envoûtement du lecteur, voir Boileau-Narcejac, Le roman policier, Paris, PUF, 1975, p. 124-125. 
Conçu autour de la séduction esthétique, le roman policier ne donne jamais tout à voir, si ce n'est peut-être à la fin, lorsque le lecteur a l'illusion de disposer des mêmes indices que le détective. Si le roman policier donnait tout à voir, il tomberait dans la même sphère que la pornographie, c'est-à-dire dans un univers unidimensionnel trop réel et trop exact. Dans le roman policier, l'auteur donne à suggérer ; dans la pornographie, tout est donné, rappelle Baudrillard, et par conséquent toute poussée transgressive liée au principe de plaisir se trouverait inhibée. Seul le désir, dans sa violence irrationnelle, "dans sa pulsion hérétique et insurrectionnelle vers la totalité peut offrir une alternative révolutionnaire $»^{6}$.

Si le roman policier appartenait à un domaine unidimensionnel hyperréaliste comparable à l'hyperréalisme pornographique, il ne contiendrait que des certitudes et nierait le désir. Or - c'est là ma première hypothèse-, dans le roman policier rien n'est certain, et parfois même la solution n'est pas complètement arrêtée. Le roman policier est l'expression d'une incertitude, d'un désir non assouvi ; il est l'expression d'une excitation de l'esprit. Dans certain cas, du moins dans ceux que nous allons examiner, le désir suscité chez le lecteur par la séduction esthétique peut quelquefois contenir une portée transgressive justement dans la frustration d'un désir non assouvi.

Fondé sur une oscillation continuelle entre logique et imaginaire, ancré dans le suspense, le roman policier ne se résume jamais, puisqu'on déflorerait le plaisir de la lecture ${ }^{7}$. Il y a du suspense dans la séduction entre un homme et une femme ; il y a une oscillation continuelle entre logique et imaginaire. Une femme ne se séduit pas deux fois, en général, à moins que l'on ne soit un adepte d'Alexandre Jardin : une fois qu'elle a été séduite, la femme devient autre. Encore faut-il qu'elle ait été séduite et non pas désignée, comme le fait l'Alligatore pour Virna, dans Nessuna cortesia all'uscita :

A servire ai tavoli ci pensava Virna. Di nome faceva Giovanna ma la chiamavamo così perché qualcuno aveva notato la sua somiglianza con Virna Lisi [...]. Quella ragazza cominciava a piacermi. [...]. Prima o poi l'avrei conquistata. Bisognava solo trovare il tempo di elaborare una strategia di corteggiamento. Magari vincente ${ }^{8}$.

6. Baudrillard J., "Le ludique et le policier ", Utopie n 2/3, mai 1969, in Le Ludique et le policier, Paris, Sens \& Tonka, 2001, p. 23-25.

7. Boileau-Narcejac, op. cit., p. 228.

8. Carlotto M., Nessuna cortesia all'uscita, Rome, E/O, 1999, p. 19. Dorénavant, l'indication des pages sera donnée directement après la citation, entre parenthèses. 
Quelque temps après, l'Alligatore et Virna ont une conversation. Ce serait le moment où l'homme pourrait déployer sa stratégie gagnante : en réalité, il perd ses moyens rhétoriques. Quant à Virna, il ne s'agit point d'une femme fatale en train de faire un strip-tease, telle Rita Hayworth dans Gilda, par exemple. Ses gants, rigoureusement noirs, ne sont pas de soie mais de caoutchouc, car elle est en train de passer la serpillière :

Virna mi chiese di farle compagnia mentre lavava il pavimento [...].

"Ce l'hai una donna? " mi chiese mentre tenevo i piedi alzati per permetterle di pulire sotto il mio tavolo.

"No, ma se hai qualche ora di tempo ti posso raccontare un paio di storie tristi e lacrimevoli. Pensa che l'ultima donna che ho amato follemente ha tentato di uccidermi un paio di volte ".

Lei rise di gusto pensando a uno scherzo. Si appoggiò al bastone dello straccio. "Anch'io non ho nessuna storia al momento ».

«Non ti ho chiesto nulla» mi affrettai a chiarire.

"Appunto. Te l'ho detto perché non me l'avresti mai chiesto » ribattè in tono pratico e poi aggiunse : « Ho pensato che potremmo metterci insieme o almeno provarci ». "Così. Senza corteggiarci. Sei proprio una romanticona ".

Virna si tolse lentamente i guanti di spessa gomma nera lunghi fino al gomito e andò al banco a preparare da bere. [...]. «Secondo me vieni sempre in questo locale perché ci sono io. Ho visto come mi guardi e son sicura di piacerti » continuò seria. "Anche tu non sei male. Magari sei uno stronzo [...] ». Virna abitava in una vecchia palazzina del centro di Padova, ristrutturata durante i meravigliosi anni Ottanta in proficui miniappartamenti da trentasette metri quadri ognuno. Scopammo con circospezione e scarsa fantasia.

«Magari la prossima volta ci scateniamo e facciamo delle cose pazzesche » disse lei alla fine, infilandosi la camicia da notte.

«Magari » approvai con entusiasmo. (p. 43-44)

Ce sera pratiquement tout. De temps en temps, les deux tourtereaux auront l'occasion de se voir et de passer une nuit ensemble, en toute pudicité, comme d'ailleurs le conseille Sciascia dans son article sur Casanova et l'utopie : "La monotona ripetizione di quelle scene per cui sono considerate di acceso erotismo $\mathrm{mi}$ ha piuttosto inclinato alla contraria manzoniana considerazione e osservanza che di amore - e di fare l'amore - al mondo ce n'è tanto che non occorre metterlo nei libri »'.

Par conséquent, bien que les romans de Carlotto soient très transgressifs et subversifs, les évocations des images d'amour et de séduction demeurent très limitées et sont finalement très classiques, du moins pour ce qui concerne l'Alligatore, qui est un ancien détenu en quête de normalité et d'un quotidien aussi banal que possible ${ }^{10}$. L'amour pour Virna

9. Sciascia L., "L'utopia di Casanova ", Cruciverba, op. cit., p. 1022.

10. Je remercie Massimo Carlotto pour avoir parlé de Virna lors de son intervention le 18 novembre 2004 à l'université de Savoie. 
donne un sens de vérité et de normalité à la vie de l'Alligatore qui, pour rien au monde, ne veut renoncer à ce sens-là dans sa vie privée.

\section{Le roman policier et la séduction sexuelle : seconde hypothèse}

Pour ce qui est du thème amoureux entre protagonistes principaux, d'ailleurs, Carlotto respecte assez les règles du genre. Il est bien connu que les histoires d'amour doivent être bannies du roman policier, du moins selon Van Dine : "Le véritable roman policier doit être exempt de toute intrigue amoureuse. Y introduire de l'amour serait, en effet, déranger le mécanisme du problème purement intellectuel $»^{11}$.

L'amour, surtout s'il concerne le détective, détournerait l'attention du lecteur et fourvoierait l'enquête : «L'amour est désordre et ne se laisse pas enfermer dans un plan " (ibid., p. 88). Du coup, dans les romans policiers classiques, il n'y a pas de véritables histoires d'amour : s'il y en a, elles sont tragiques - songeons à 120 rue de la gare, de Léo Malet - ou bien réduites à l'essentiel. Maigret aime Mme Maigret sans frémissements, sans excès sensuels et presque sans ennui : une présence asexuée et inutile, en apparence. Cependant, comme l'écrivait Michel Décaudin, Mme Maigret est nécessaire, sans elle Maigret "ne serait plus tout à fait Maigret " ${ }^{12}$.

Pourtant, il y a dans certains polars des histoires de séduction entre le détective et une femme - nous parlerons ici de séduction sexuelle - et qui servent en général à trahir et à confondre le lecteur. Par ailleurs, l'évolution de la séduction sexuelle coïncide, souvent, avec la relation de séduction esthétique : là où il y a jeu de séduction entre personnages - voici ma seconde hypothèse - on peut trouver souvent des indices utiles à la solution de l'enquête.

\section{Fruttero \& Lucentini, ou l'adultère transgressif}

La donna della domenica est un roman policier des plus classiques. Le jeu de séduction entre Anna Carla - une femme mariée - et le commissaire

11. Cité in Boileau-Narcejac, op. cit., p. 97.

12. Décaudin M., "Enquête sur une personne au-dessus de tout soupçon ", p. 387 du tiré à part. M. Décaudin m'avait envoyé cet article quelques semaines avant de décéder. Il n'est plus là pour répondre à toutes les questions que j'avais encore à lui poser, il n'est plus là pour encourager certains projets et il nous a laissé un vide impossible à combler. Et il n'est plus là non plus pour me dire quand et où il avait publié ce texte sur Mme Maigret. 
Santamaria - célibataire - est exemplaire : au fur et à mesure que l'enquête avance, le jeu de la séduction sexuelle se fait de plus en plus serré. Néanmoins, ce n'est qu'à la toute dernière page que l'on découvre les deux personnages au lit, en train non pas de s'amouracher, mais de faire le point sur l'histoire policière :

- Ho telefonato in questura. Forse non era tanto il momento, ma non resistevo più. Morivo letteralmente dalla voglia di...

Non era di quella voglia, che moriva. Quella voglia se l'era levata dopo, bevendo Coca-Cola, spostando cuscini, fumando, perdendo accendini, facendo molte domande, sì, chiedendo molti particolari. Ma non poi troppi, si disse il commissario ottimisticamente, non poi così insistenti, minuziosi.

- Che ora sarà?

[...] Il commissario accese la luce, guardò l'orologio.

- Sono le sette e venti.

- Oh mipovradona! disse ridendo Anna Carla. Ma è tardissimo !

Scese dal letto leggera, e cominciò in fretta a rivestirsi ${ }^{13}$.

Dans $A$ che punto è la notte $e^{14}$, nous retrouvons le même commissaire Santamaria aux prises avec une sorte de sœur jumelle d'Anna Carla, $\mathrm{M}^{\mathrm{me}} \mathrm{Guidi}$, également mondaine, désœuvrée et mariée.

Le commissaire et la belle dame se rencontrent au commissariat. Guidi Costanza - pour la première et dernière fois le prénom de la dame est explicité - est interrogée suite à la mort de Don Pezza. Tout d'abord, Santamaria pense qu'il s'agit d'une prostituée (p. 234) ; puis elle devient " bella mamma " à la fin de l'interrogatoire, lorsqu'elle explique sa situation et surtout celle de sa fille, Thea, dont le petit ami est un mafieux. C'est dans le journal de l'agent Pietrobono que nous obtenons d'autres indices sur le jeu de séduction en cours, ainsi que sur certains mouvements à l'intérieur du commissariat. La séduction esthétique intervient alors pour maintenir en haleine le lecteur, tout en l'amusant : "Bella C.G., devo ulteriorm. dire, non convincermi troppo, anzi, stammi abbast. sulle scat. - Suo gran cine (di cui accortami beniss.) per incantare celebre S.maria (che cascatoci come cret.) [...]» (p. 239).

Dès que le commissaire tâtonne, dès que l'enquête arrive à un point mort, les indices de la séduction sexuelle viennent dynamiser la situation ; dès que la séduction sexuelle est mise en place, l'enquête momentanément essoufflée retrouve son élan. Comme dans La donna della domenica, le cas

13. Fruttero \& Lucentini, La donna della domenica (1972), Milan, Mondadori, 1989, p. $502-$ 503.

14. Fruttero \& Lucentini, A che punto è la notte (1979), Milan, Mondadori, 1993. Dorénavant, l'indication des pages sera donnée directement après la citation, entre parenthèses. 
sera complètement résolu lorsque Santamaria et la femme seront physiquement très proches. Mme Guidi se glissera alors dans le lit avec le commissaire, plus ou moins de la même manière qu'Anna Carla.

Le retardement de la révélation complète du dénouement fait partie de la stratégie de séduction esthétique qui accompagne le jeu de séduction sexuelle entre Mme Guidi et Santamaria : «Ma dov'era intanto, si stavano chiedendo in parecchi, il commissario Santamaria ? Perché tardava ? Perché non si faceva trovare ? Perplessità di ogni genere crescevano attorno alla sua inspiegabile assenza " (p. 598). Même le Grande Boss, qui a pris la relève du narrateur pour l'accomplissement de certaines tâches, le cherche partout : "Il Grande Boss, che conosceva il suo uomo, non ci mise molto a localizzarlo, né si stupì di vederlo impegnato in una conversazione di alto tenore intellettuale e morale» (p. 599).

La dernière conversation entre Santamaria et la signora Guidi peut être lue tantôt comme un commentaire à l'histoire de Santa Liberata, tantôt comme un commentaire à l'adultère sur le point d'être consommé :

- Ma io non sono mica gnostica, non sono mica infame ! - si ribellò la signora Guidi giocherellando con un elastico. - Io continuo a trovare che la vecchia dottrina, i vecchi divieti, il vecchio contra sextum...

Ora non aveva più niente addosso. S'infilò per prima sotto le diacce lenzuola della stanza $n^{\circ} 12$.

- Perché insomma, - disse con un brividino, - il vecchio Dio, con tutti i suoi difetti...

Il Grande Boss chiuse l'altro occhio. (p. 601)

L'autre œil, le Grande Boss l'avait déjà fermé pour ne pas avoir à regarder Thea et Graziano en train de faire l'amour en voiture. Les yeux du Grande Boss se ferment, pour une fois et pour une nuit. Le cas est résolu, on n'a plus besoin de maîtriser la rationalisme et on peut laisser libre cours, enfin, à la sensualité, à l'amour, à l'indétermination et l'imagination.

Il faut remarquer tout de même qu'il y a, dans ce roman, des histoires d'amour secondaires, comme celle entre Thea, la fille de Mme Guidi, et Graziano, le mafieux : quand Thea s'aperçoit qu'elle est amoureuse, elle bascule de la Verführung au Zauber ${ }^{15}$, du détournement implicite de la séduction à la magie. Dès lors se met en place un univers à part, où le méchant mafieux devient bon, collabore avec la police et trouve des indices déterminants pour la solution de l'enquête. Le couple TheaGraziano est très différent du couple signora Guidi-Santamaria. Il y a

15. Schérer R., «La composition du charme », (8 octobre 2003) :

$<$ http://raforum.apinc.org/imprimerart.php3?id_article=1636>, 
même presque opposition : le premier couple, en effet, passe de l'indétermination sentimentale au déterminisme amoureux; le second bascule du déterminisme décisionnel pré-séductifà l'indéterminisme sensuel contenu dans l'adultère. Autrement dit, le couple Thea-Graziano va vers la révélation de l'intrigue, alors que le couple Signora Guidi-Santamaria éloigne le lecteur de la solution.

\section{Comme «Un disco dei Platters "}

Le jeu de séduction apparaît évident dans Un disco dei Platters ${ }^{16}$, de Macchiavelli et Guccini. Le protagoniste, détective un peu malgré lui puisqu'il est censé être en vacances, est le maresciallo Santovino. À la fin des années $50^{17}$, Santovino éprouve l'envie de revoir le village des Apennins où il avait travaillé dans sa jeunesse ${ }^{18}$. Pendant le voyage, le sympathique quinquagénaire ou présumé tel fait la connaissance d'une jeune femme, Raffaella Anceschi, qui se rend dans le même village pour des raisons professionnelles.

Comme dans $A$ che punto è la notte, l'histoire de séduction sexuelle procède au même rythme que la séduction esthétique : Santovino interroge Raffaella sur sa vie privée alors qu'ils sont en train de discuter du trésor de la Regina Selvaggia (p. 115); elle l'appelle pour la première fois Benedetto (p. 117), au moment où ils doivent monter à l'Abbaye afin de poursuivre leurs recherches. Les deux personnages s'approchent de plus en plus : le jeu de séduction devient à la fois attraction inéluctable et prétexte nécessaire au développement de l'histoire policière.

Santovino est plutôt passif, cependant : c'est Raffaella qui l'embrasse la première sur la joue (p. 165), c'est elle qui prend l'initiative et mène la danse du jeu de séduction. Lorsque Santovino s'en aperçoit, un peu tardivement, il essaie de protester, comme s'il se sentait piégé. L'apparition de Cleto, lors d'une discussion entre eux deux, est providentielle, du moins pour Santovino et pour l'enquête :

16. Macchiavelli \& Guccini, Un disco dei Platters, Milan, Mondadori, (1998), 1999. Dorénavant, l'indication des pages sera donnée directement après la citation, entre parenthèses.

17. "Un disco dei Platters" est évidemment une chanson écrite et interprétée par le Quartetto Cetra dès 1957 et «Nel blu dipinto di blu » cité à la fin du roman gagne le Festival de Sanremo en 1958.

18. Les débuts de la carrière de Santovino sont racontés dans Macchiavelli \& Guccini, Macaroni, Milan, Mondadori, 1997. 
"Ma santamadonna, possibile che non ti accorgi... Ooh, io sono un uomo e tu una donna!»

"Lo so. Per questo stiamo bene assieme, non ti pare?"

"Insomma, mi ricevi in casa tua di sera tardi, mi fai certi discorsi... Mi baci e, santamadonna!, non sono tuo padre!»

Il viso di Raffaella si rilassò. Sorrise: «Ah, solo per questo ! Io chissà che credevo ! Lo so che non sei mio padre [...]».

Santovino uscì dalla stanza e Raffaella lo seguì :

«No, senti, Benedetto...» Da anni non era Benedetto : Santovino per tutti e sentirsi chiamare con il nome di battesimo... "Non pensare che io... Insomma, cerca di capire...»

Santovino posò la valigia sul primo gradino per lasciare libere le mani di agitarsi a loro piacere, come non faceva da tempo : "Cosa devo pensare ? E che c'è da capire? Se me lo spieghi...»

Lo interruppe Cleto, rosso in viso [...]. (p. 166-167)

Le jeu des regards, les atermoiements, sont autant d'éléments qui contribuent au prolongement du suspense et donc au retardement de l'inévitable premier baiser sur la bouche (p. 199), échangé lorsque Raffaella propose à Santovino une tentative d'explication de la mort du Romitto. L'enquête policière, basée sur la quête des indices - autant de signes laissant prévoir d'autres circonstances incertaines ou à venir ${ }^{19}$ - est très proche du jeu de séduction sexuelle. Et la supercherie a un rôle fondamental dans les deux cas : ce qui est donné à voir ne correspond jamais tout à fait à la vérité. Si c'était le cas, d'ailleurs, le lecteur se retrouverait comme plongé dans le discours interprétatif, qui est « le moins séduisant qui soit $»^{20}$.

Ce n'est que plus tard et plus ou moins à cause d'un orage que Raffaella demande - ou plutôt exhorte - Benedetto à passer la nuit avec elle. Après cette nuit, évidemment écourtée pour les raisons de l'enquête, Raffaella et Santovino deviennent d'inséparables complices, mais la séduction et la sensualité ont disparu. Raffaella est devenue le faire-valoir du détective : comme Holmes et Watson, Poirot et Hastings, Santovino et Raffaella deviennent complémentaires, même si, évidemment, c'est lui qui trouve la solution, après avoir quitté la jeune femme. En effet, Santovino quitte le village à la fin des vacances, et Raffaella, telle Didon abandonnée par Énée, est accablée ${ }^{21}$; elle lui demande de revenir vite et Santovino ne répond pas. L'affaire amoureuse n'est pas du tout résolue, et l'affaire judiciaire non plus : dans le train, Santovino retrouvera ses esprits, ce qui permettra enfin au lecteur de connaître au moins la fin de l'histoire policière.

19. Grimaldi L., Il giallo e il nero, Milan, Pratiche Editrice, 1996, p. 26.

20. Baudrillard J., De la séduction, Paris, Denoël, 1979, p. 76.

21. Merci à Loriano Macchiavelli de m'avoir livré cette suggestion. 


\section{De la séduction féminine à l'attraction partagée}

Je ne pourrai pas effectuer une analyse approfondie de l'image de la séduction dans La collega tatuata de Margherita Oggero, qui se situe dans la lignée de Fruttero \& Lucentini : le commissaire - Gaetano -, que la professoressa trouve belloccio, a les ongles propres, sait conjuguer le subjonctif, et est par conséquent un héritier du commissaire Santamaria ${ }^{22}$. La professoressa s'amourache de lui, imperceptiblement, dans son flux de conscience ( " perché lo tratto così confidenzialmente sia pure soltanto nel mio flusso di coscienza ?., p. 76) et essaie de le séduire avec la parole, en partie aussi pour lui arracher des informations utiles à l'enquête en cours :

Oddio, forse mi sta facendo la corte. $\mathrm{O}$ forse mi sfotte [...]. Di sicuro invece io sto civettando. Senza esibire tette e culo (che non sono un granché), senza protendere labbroni siliconati da sesso presidenziale, senza sbattere ciglia da giraffa (sono più lunghe di quelle da cerbiatta [...]), ma con le parole, che è il modo che più mi piace, anzi l'unico che mi piace veramente. La semplice arte del delitto come anticipazione di un eccitante marivaudage. (p. 112)

L'art de la séduction, associé ici à l'art du délit - en papier - demeure une affaire de logos. Gaetano réagit favorablement à la séduction sexuelle et le lecteur cède à son tour à la séduction esthétique : c'est bien lors des rencontres entre Gaetano et la professoressa que le lecteur obtient des informations sur l'enquête. "Sto cacciandomi in una storia che ha come sbocco prevedibile l'adulterio" (p. 168), se dit-elle au fur et à mesure que l'enquête avance. Brusquement, elle arrête le jeu de séduction sexuelle : Gaetano et elle deviennent amis et complices, comme l'ont été Raffaella et Santovino. Cependant la professoressa sans nom n'est pas le faire-valoir du détective, parce qu'elle est elle-même détective : du coup, elle parvient à la solution grâce à son flair, en même temps que Gaetano (p. 234).

\section{La séduction transgressive}

La relation entre Grazia Negro et Simone, dans Almost Blue, est très étrange. Ici, ce n'est pas Grazia qui séduit l'aveugle Simone, mais c'est Simone qui est séduit par Grazia, sans qu'elle s'en rende compte. Pour lui, elle est tout d'abord une voix ${ }^{23}$, puis une voix bleue dans un rêve (p. 86),

22. Oggero M., La collega tatuata, Milan, Mondadori, 2002, p. 66 ; dorénavant, l'indication des pages sera donnée directement après la citation, entre parenthèses.

23. Lucarelli C., Almost Blue, Turin, Einaudi, 1997, p. 39 ; dorénavant, l'indication des pages sera donnée directement après la citation, entre parenthèses. 
une odeur qui est une musique - Grazia utilise un parfum qui s'appelle Summertime (p. 96). C'est pour cette raison que Simone accepte de participer à l'enquête et d'aider Grazia : il a peur de ne plus entendre sa voix et de ne plus sentir son odeur.

La séduction que Grazia exerce à son insu sur Simone est entièrement fondée sur la parole, la voix, les sons, puis aussi sur le toucher et les odeurs (p. 176). Lorsque la séduction se transforme en attraction réciproque (comme dans le cas de Raffaella et Santovino), Grazia devient Holmes et Simone devient Watson ; comme Raffaella, Simone est le complémentaire indispensable au développement de l'enquête.

Dans leur relation, cependant, le passage de la séduction à la magie d'amour n'a plus du tout la force de transformer la solution de l'affaire en dénouement heureux. D'où évidemment l'apparition de la transgression chez Lucarelli : après avoir pris les traits de Simone, l'Iguana pourrait encore s'échapper et tuer; à la fin du roman, Simone a une musique qui résonne dans la tête, comme c'était le cas pour l'Iguana ; Grazia, qui est censée être la détective inébranlable, apparaît constamment dans toute sa fragilité de femme. Et surtout, dans le dernier chapitre d'Almost Blue, il y a une tension vers un futur plus ou moins prévisible et très inquiétant : alors qu' un polar de Fruttero \& Lucentini se terminerait sur un happy end où le couple commente au lit l'enquête et donne au lecteur les derniers détails, ici, Grazia et Simone ne sont pas encore au lit et la séduction esthétique, par conséquent, laisse le lecteur dans une situation de désir insatisfait du fait de l'insolubilité du cas. La séduction sexuelle n'a pas abouti complètement. Quant à la séduction esthétique, non seulement elle n'a pas amené de vérité absolue, mais elle a contribué à l'écarter : retrouvant ici sa signification étymologique originelle, la séduction soustrait et détourne la vérité. Nous avons alors une illusion de la fin-encore une allusion fugace à Baudrillard ${ }^{24}$ - où non seulement le crime est imparfait, mais où l'écartement du droit chemin nous amène au centre de l'extrême et au bord de l'impensable.

24. Il n'est évidemment pas question, ici, de faire référence à tout le discours de Jean Baudrillard sur le concept d'extermination de l'illusion et du réel contenu dans son ouvrage Le crime parfait, Paris, Galilée, 1995 ; voir aussi Baudrillard J., L'illusion de la fin, Paris, Galilée, 1992. 


\section{L'illusion de la fin}

Toute histoire policière devrait rétablir un équilibre entre le Bien et le $\mathrm{Mal}$, ce qui n'est pas le cas chez Lucarelli. Il n'y a plus aucun équilibre à rétablir, d'ailleurs, parce que la société que nous nous sommes construite n'a jamais été en équilibre. La consolation mystificatrice n'existe plus.

Si la vérité n'est qu'une illusion, c'est que toute apparence cache une supercherie, comme la séduction, du reste, qui ne serait qu'un jeu de brouillage des pistes parmi tant d'autres. D'où l'inquiétude constante véhiculée par le roman policier italien contemporain ; d'où également l'inquiétude constante qui nous accable dans la réalité, ou dans l'illusion de la réalité. En même temps, comme dans un roman policier, nous ne sommes pas capables de nous soustraire à cette illusion inquiétante de la fin qui nous charme et nous envoûte.

LA ROMANCIÈRE (au lecteur)

Ceci est une histoire vraie, je vous le jure. Oh, j’ai changé les noms, bien sûr ; j’ai changé les lieux, l'époque, [...], les dialogues, l’ordre des événements [...] ; et pourtant, tout ce que je vais vous raconter est vrai. C'est [...] une fantasmagorie comme toujours : les témoins vont converger ici et s'efforcer un à un de vous convaincre, de vous éblouir, de vous mener en bateau $[\ldots]^{25}$.

En somme, le cercle ne peut pas se clore, du fait que la vie imite la littérature et que la littérature imite la vie. Il n'y a pas de solution à cette dialectique, qui n'est qu'en apparence une aporie.

25. Huston N., Une adoration, Paris, Actes Sud, 2003, p. 11. 